\title{
ON THE CLASSIFICATION OF LIE ALGEBRAS OF PRIME CHARACTERISTIC ${ }^{1}$
}

\author{
BY JOHN B. JACOBS
}

Communicated by George B. Seligman, August 25, 1969

This paper presents results on the classification of finite-dimensional simple Lie algebras of prime characteristic. Unlike the simple algebras of characteristic zero, these algebras do not necessarily possess a nondegenerate trace form-an important tool in classifying the characteristic zero algebras. Those which do possess such a form have been classified by G. B. Seligman [9] and, not unexpectedly, have been found to be analogs of the simple algebras of characteristic zero. However, further large classes of a quite different nature exist. Having no characteristic zero analogs, they are said to be of nonclassical type. Important work on the classification of such algebras has been done by A. I. Kostrikin ([5] and a long series of earlier papers) and by Kostrikin and I. R. Safarevič ([7] and [8]). In an attempt to provide a uniform classification theory, they have considered the infinite Lie algebras over the complex numbers (see [1], [2], [3]). These algebras are said to be of Cartan type and have been completely classified. By replacing the complex numbers in the construction by an algebraically closed field of prime characteristic, Kostrikin and Šafarevič have produced a unified way of describing all the known nonclassical simple restricted Lie algebras. This has been further generalized by R. L. Wilson [10] to include all the known simple algebras of nonclassical type. The fact that their construction exhausts the known simple restricted algebras has led Kostrikin and Safarevič to conjecture that there are no more such algebras. This conjecture has been proved in a very special case [7, Theorem 1]. As has developed in their work, the classification problem splits into two parts: proving the existence of a long filtration and using the filtration in an attempt to classify the algebras. By a filtration of a finite-dimensional Lie algebra $L$ is meant a chain of subalgebras

$$
L=L_{-1} \supset L_{0} \supset \cdots \supset L_{r} \supset L_{r+1}=0,
$$

AMS Subject Classifications. Primary 1730.

Key Words and Phrases. Classification of simple Lie algebras, strongly degenerate, $H$-uniform elements, long filtration, nilpotent elements, irreducible representation.

${ }^{1}$ This research is contained in the author's doctoral dissertation written under the direction of Professor Richard E. Block at the University of Illinois, Urbana, and the University of California, Riverside. 
where $L_{0}$ is an arbitrary proper subalgebra (usually taken to be maximal) and the remaining subalgebras are defined inductively by $L_{i+1}=\left\{x \in L_{i} \mid[x, L] \subset L_{i}\right\}$. A filtration is said to be long if $r \geqq 2$.

The present paper is a contribution toward establishing the existence of a long filtration in the simple Lie algebras which are not of classical type. Kostrikin [5] has shown this to be equivalent to an algebra $L$ being strongly degenerate, that is, possessing an element of index of nilpotence two. An element $x$ in $L$ with $L(\operatorname{ad} x)^{k}=0$, $L(\operatorname{ad} x)^{k-1} \neq 0$ is said to be nilpotent of index $k$. Kostrikin's main result in [5] is formulated as follows. If $L$ is a simple Lie algebra over an algebraically closed field of characteristic $p>5$ which satisfies:

(i) $L$ is restricted, that is, ad $L$ is closed under $P$ th powers (restricted Lie algebras are also called Lie $p$-algebras),

(ii) $L$ is not strongly degenerate,

(iii) $L$ has a Cartan subalgebra $H, L=H+\sum_{\alpha \neq 0} L_{\alpha}$, for which $L(\operatorname{ad} a)^{p-1}=0$ for some nonzero $a$ in $H$ or in some root space $L_{\alpha}$, then $L$ is of classical type.

If, as in (iii), an element $a$ is either in $L_{\alpha}$ for some $\alpha$ or in $H$, it is called $H$-uniform. In addition to weakening (ii), we can show that deleting (i) does not alter the conclusion. Our first main result is the following.

THeOREM A. Let $L$ be a simple algebra over an algebraically closed field $F$ of characteristic $p>5$. Let $H$ be a Cartan subalgebra of $L$ for which (iii) is satisfied, and further suppose that any nilpotent $H$-uniform element has index of nilpotence greater than two. Then L is of classical type.

We remark that condition (iii) cannot be deleted from the hypothesis of Theorem A as the nonclassical p-dimensional Witt algebra possesses a Cartan subalgebra for which no uniform element is nilpotent.

This theorem is a contribution toward showing (as Kostrikin conjectures) that strong degeneration differentiates between algebras of classical and nonclassical type (it can be easily shown that no classical algebra is strongly degenerate).

We now consider some special algebras of low dimension relative to $p$. Suppose $L$ is a simple Lie algebra over an algebraically closed field of characteristic $p>5$. Kostrikin has shown that $L$ is of classical type if $\operatorname{dim} L<p$, and he has some partial results [4] for dimension $p$. We use Theorem A and several results of Kostrikin to show that if $L$ has dimension $p$, then it is either the nonclassical $p$-dimensional Witt algebra or of classical type (Theorem B). The final theorem, which proves a conjecture of Kostrikin in [6], is the following. 
TheORem C. Suppose L is a semisimple Lie algebra over an algebraically closed field $F$ of prime characteristic which admits a faithful representation $\Gamma$ of degree $n<p-1$. Then $L$ is a direct sum

$$
L=L_{1} \oplus \cdots \oplus L_{r},
$$

where each $L_{i}$ is a simple algebra of classical type.

Kostrikin has proved this under the additional hypotheses that $L$ is a $p$-algebra and $\Gamma$ is a $p$-representation.

\section{REFERENCES}

1. E. Cartan, Les groupes de transformations continus, infinis simples, Ann. Sci. Ecole Norm. Sup. 26 (1909), 93-161.

2. V. W. Guillemin and S. Sternberg, An algebraic model of transitive differential geometry, Bull. Amer. Math. Soc. 70 (1964), 16-47. MR 30 \#533.

3. S. Kobayashi and T. Nagano, On filtered Lie algebras and geometric structures. II, J. Math. Mech. 14 (1965), 513-521. MR 32 \#2512.

4. A. I. Kostrikin, Simple Lie p-algebras, Trudy Mat. Inst. Steklov. 64 (1961), 79-89; English transl., Amer. Math. Soc. Transl. (2) 55 (1966), 194-206. MR 24 \#A1933.

5. - Squares of adjoint endomorphisms in simple Lie p-algebras, Izv. Akad. Nauk SSSR Ser. Mat. 31 (1967), 445-487= Math. USSR Izv. 1 (1967), 435-473. MR 36 \#1501.

6. — Theorem on semisimple Lie p-algebras, Mat. Zametki. 2 (1967), 465474. (Russian) MR 36 \#1502.

7. A. I. Kostrikin and I. R. Safarevič, Cartan's pseudogroups and the p-algebras of Lie, Dokl. Akad. Nauk SSSR 168 (1966), 740-742=Soviet Math. Dokl. 7 (1966), 715-718. MR 33 \#7384.

8. - Graded Lie algebras of finite characteristic, Izv. Akad. Nauk SSSR Ser. Mat. 33 (1969), 251-321= Math. USSR Izv. 3 (1969) (to appear).

9. G. B. Seligman, On Lie algebras of prime characteristic, Mem. Amer. Math. Soc. No. 19 (1956). MR 17, 1108.

10. R. L. Wilson, Nonclassical simple Lie algebras, Bull. Amer. Math. Soc. 75 (1969), 987-991.

UNIVERSITY OF ILLINOIS, URbaNa, ILlinoIs 61801 aND

University of California, Riverside, California 92502 